[6] R. E. Curto, Fredholm and invertible tuples of operators. The deformation problem, Trans. Amer. Math. Soc. 266 (1981), 129-159.

[7] R. E. Harte, Invertibility, singularity and Joseph L. Taylor, Proc. Roy. Irish Acad. Sect. A 81 (1981), 399-406.

[8] -, Fredholm, Weyl and Browder theory, ibid. 85 (1985), 151-176.

[9] -, Invertibility and Singularity for Bounded Linear Operators, Dekker, New York, 1988.

10] -, Index continuity for chains, in: Aportaciones Matematicas en Memoria del Profesor Victor Manuel Onieva Aleixandre, Univ. de Cantabria, Santander, 1991, 199208; MR 92f:47011.

[11] -, Taylor exactness and Kato's jump, Proc. Amer. Math. Soc. 119 (1993), 793-802.

12] M. Putinar, Some invariants for semi-Fredholm systems of essentially commuting operators, J. Operator Theory 8 (1982), 65-90.

[13] J. L. Taylor, A joint spectrum for several commuting operators, J. Funct. Anal. 6 (1970), 172-191.

[14] F.-H. Vasilescu, A characterization of the joint spectrum in Hilbert space, Rev. Roumaine Math. Pures Appl. 22 (1977), 1001-1009.

15] -, On pairs of commuting operators, Studia Math. 62 (1978), 203-207.

[16] -, Stability of the index of a complex of Banach spaces, J. Operator Theory 2 (1979), 247-275.
SCHOOL OF MATHEMATIOS

TRINITY COLLEEGE

DUBLIN 2, IRELAND

E-mail: RHARTE@MATHS.TCD.IE
DEPARTMENT OF MATHEMATICS SUNG KYUN KWAN UNIVERSITY SUWON 440-746, KOREA E-mail: WYLEEQYURIM.SKKU.AC.KR

\section{A remark on non-existence of an algebra norm for the algebra of continuous functions \\ on a topological space admitting an unbounded continuous function}

by

ATEXANDER R. PRUSS (Vancouver)

Abstract. Let $X$ be any topological space, and let $C(X)$ be the algebra of all continuous complex-valued functions on $X$. We prove a conjecture of Yood (1994) to the that if there exists an unbounded element of $C(X)$ norrned algebra.

Throughout, $C(X)$ denotes the algebra of all continuous complex-valued functions on a topological space $X$.

TrEOREM. Let $X$ be a topological space such that $C(X)$ has an unbounded element. Then there is no normed algebra norm on $C(X)$.

As Yood [4] notes, it is easy to see that there is no Banach algebra norm on $C(X)$, so that the main content of the Theorem is the non-existence of a norm under which $C(X)$ would be an incomplete normed algebra. Our Theorem was conjectured by Yood [4] who showed that it does hold under the additional assumption that $X$ is a locally compact Hausdorff space such that every character of $C(X)$ is a point-evaluation. Yood also showed that this condition on the characters is implied by the existence of a function $h \in C(X)$ such that $\{x: h(x)=\alpha\}$ is compact for every $\alpha \in \mathbb{C}$.

To prove our Theorem, we recall a result due to Kaplansky [2, Thm. 6.2] and also used by Yood [4] in his work. By $C_{0}(X)$ we mean the algebra of continuous complex-valued functions on $X$ vanishing at infinity, where $X$ is a topological space and $C_{0}(X)$ is always equipped with the supremum norm. For more information on interesting issues related to the following Proposition, see [3, pp. 244 and 576-579].

199.1 Mathematics Subject Classification: 46E25, 46J10

Key words and phrases: algebra of all continuous functions, normed commutative algebra, non-existence of norm, topological spaces admitting unbounded functions. 
Proposition (Kaplansky). Let $(A,\||\|\mid\|)$ be a commutative Banach algebra which is isometrically isomorphic to $C_{0}(X)$ for some locally compact $X$. Then any normed algebra norm $\|\cdot\|$ on $A$ satisfies

$$
\|f\| \geq\|f\|
$$

for every $f \in A$.

Now, let $B(X)$ denote the algebra of bounded continuous complex-valued functions on a topological space $X$.

Corollary of Proposition. Let $X$ be any topological space and let $\|\cdot\|$ be a normed algebra norm on $B(X)$. Then for every $f \in B(X)$ we have

$$
\|f\| \geq \sup _{x \in X}|f(x)| .
$$

Proof of Corollary. By [3, Thm. 3.2.12], the algebra $B(X)$ equipped with supremum norm is isometrically isomorphic to $C_{0}(\Gamma)$, where $\Gamma$ is its maximal ideal space. Since $\Gamma$ is locally compact [3, Thm. 3.1.2], the Corollary follows from the Proposition.

Pro of of $\mathrm{T}$ heorem. Suppose $\|\cdot\|$ is a normed algebra norm on $C(X)$. Then it is also a normed algebra norm on $B(X)$, and by the Corollary given above it follows that

$$
\|F\| \geq \sup _{x \in X}|F(x)|
$$

for any $F \in B(X)$. Now, if a function $h \in C(X)$ vanishes nowhere on $X$ then we write $h^{-1}$ for its reciprocal. Clearly $h^{-1}$ is also in $C(X)$ then. We write 1 for the function which is identically 1 on $X$. By assumption, there is an unbounded continuous complex-valued function $g$ on $X$. Let $f=\mathbf{1}+|g|$. Then $f$ is unbounded and continuous as well. Set $u=f^{-1}$. Then, since $f \geq 1$ everywhere on $X$, it follows that $u$ is bounded. Moreover, $u$ is strictly positive on $X$. Note that for any fixed $t>0$, the function $u+t \cdot 1$ is bounded on $X$ and is everywhere greater than $t$. Thus, $(u+t \cdot 1)^{-1}$ exists and is bounded. Then by $(*)$ we have

$$
\left\|(u+t \cdot \mathbf{1})^{-1}\right\| \geq \sup _{x \in X}\left|(u(x)+t)^{-1}\right|
$$

for $t>0$. (The argument from this point on has been streamlined by the referee, to whom the author's thanks are due.) Now, inversion in any normed algebra with identity is continuous on the invertible elements [1, Prop. 6 , p. 11]. Hence, $\left\|\left(u+n^{-1} 1\right)^{-1}-u^{-1}\right\| \rightarrow 0$ as $n \rightarrow \infty$, so that

$$
\sup _{n}\left\|\left(u+n^{-1} 1\right)^{-1}\right\|=M<\infty \text {. }
$$

Therefore, by $(* *)$, we have $\left|\left(u(x)+n^{* 1}\right)^{-1}\right| \leq M$ for each $x \in X$ and every positive integer $n$. But for all $x \in X$,

$$
\lim _{n \rightarrow \infty}\left(u(x)+n^{-1}\right)^{-1}=(u(x))^{-1}
$$

since inversion is continuous on $\mathbb{C} \backslash\{0\}$. Thus, $\left|(u(x))^{-1}\right| \leq M$ for every $x \in X$. This contradicts the assumption that $u^{-1}=f$ is an unbounded function.

\section{References}

[1] F. F. Bonsall and J. Duncan, Complete Normed Algebras, Springer, 1973

2. I. Kaplansky, Normed algebras, Duke Math. J. 16 (1949), 399-418.

[3] T. W. Palmer, Banach Algebras and the General Theory of $*$-Algebras. Volume I Algebras and Banach Algebras, Encyclopedia Math. Appl. 49, Cambridge Univ.
Press, 1994.

[4] B. Yood, On the nonexistence of norms for some algebras of functions, Studia Math. 111 (1994), 97-101.

\section{DEPARTMENT OF MATTHEMATICS}

UNIVERSTTY OF BRITISH COLUMBIA

VANCOUVER, B.C.

CANADA VG'C $1 Z 2$

E-mail: PRUSSOMATH.UBC.CA

Received February 16, 1995

Revised version June 16,1995

Editorial note: See also the paper by Michael J. Meyer in this issue. 